\title{
A Systematic Literature Review of the Use of Social Media for Business Process Management
}

\author{
Jana Prodanova ${ }^{1(\varpi)}$ and Amy Van Looy ${ }^{2}$ \\ ${ }^{1}$ Department of Economics and Business Administration, Faculty of Economics and Business, \\ University of Burgos, C/Parralillos, s/n, 09001 Burgos, Spain \\ jprodanova@ubu.es \\ 2 Department of Business Informatics and Operations Management, Faculty of Economics \\ and Business Administration, Ghent University, Tweekerkenstraat 2, 9000 Ghent, Belgium \\ Amy. VanLooy@uGent. be
}

\begin{abstract}
In today's expansion of new technologies, innovation is found necessary for organizations to be up to date with the latest management trends. Although organizations are increasingly using new technologies, opportunities still exist to achieve the nowadays essential omnichannel management strategy. More precisely, social media are opening a path for benefiting more from an organization's process orientation. However, social media strategies are still an under-investigated field, especially when it comes to the research of social media use for the management and improvement of business processes or the internal way of working in organizations. By classifying a variety of articles, this study explores the evolution of social media implementation within the BPM discipline. We also provide avenues for future research and strategic implications for practitioners to use social media more comprehensively.
\end{abstract}

Keywords: Business process management $\cdot$ Lifecycle management Social BPM $\cdot$ Collaborative BPM $\cdot$ Social media $\cdot$ Business 2.0

\section{Introduction}

Nowadays, social media are one of the most important instruments to enhance the information flows and relationships between individuals and organizations. The main reasons for using social media in businesses are, among others, customer satisfaction, loyalty, engagement and sales increase [1]. Contributing to external and internal business objectives, a social media strategy affects the employees, internal communication, product/ service innovation, growth related to people capabilities, systems and organizational procedures, and the optimization and management of business processes or the internal way of working in organizations [2]. As such, social media management and business process management (BPM) are closely related disciplines.

While the current body of knowledge recognizes the uptake of social media in society $[3,4]$, a lack of research is perceived related to the impact of social media on business processes and BPM. To fill this gap, this study provides a Systematic Literature Review (SLR) of the social media use by organizations, and particularly their complementarity 
with BPM. Our objective is to shed light on the state of the research on social media use in the BPM discipline and to present opportunities for future academic research and relevant recommendations for management practitioners.

Subsequently, Sect. 2 provides the theoretical background. The SLR research method is described in Sect. 3. Afterwards, the results are presented in Sect. 4 and discussed in Sect. 5, offering implications of scientific and applied relevance.

\section{Theoretical Background}

\subsection{Theoretical Background of Social Media Use by Organizations}

Since social media have a key position in the $\mathrm{B} 2 \mathrm{C}$ communication, they are implemented in the traditional CRM (Customer Relationship Management) systems [6]. Additionally, it was confirmed that new technologies affect operational and management processes as well $[6,7]$. Moreover, it is not required to use all social media tools, but rather the most suitable ones depending on corporate objectives and strategies [2, 5].

Various attempts explain the structure and purposes of social media. Most social media classifications are, however, customer-oriented [5, 8]. In addition to the more customer-oriented classifications, other classifications exist that consider implementation aspects of social media for organizations $[9,10]$. One of the most complete classifications is the honeycomb model by Kietzmann et al. [10]. This framework not only focuses on the functional characteristics of social media tools, but also on the business implications. It is represented by a honeycomb with seven blocks. First, the (1) "identity" block refers to the extent to which users reveal personal information and subjective information (e.g. opinions), which is why companies have to control data privacy and security. The functional block related to (2) "conversations" is explained by the communication between social media users, so organizations can follow conversations on a certain topic. The (3) "sharing" block represents users who exchange content or are connected by a shared object (e.g. discount vouchers). This block calls for content management systems and building social graphs for business intelligence reasons. The (4) "presence" functional block refers to the extent to which users know about other users (e.g. their location or availability). The (5) "relationships" function denotes which users are related to each other and how (i.e. the structural and flow properties in a network), which does not require a formal relationship. The (6) "reputation" functional block offers users the possibility to identify their and others' reputation based on usergenerated information, such as the number of followers or shares. Also sentiment analysis is implied in this block. Finally, the (7) "groups" block refers to the users' ability to form communities. This can be realized through membership rules and protocols.

Although studies acknowledge the usefulness of social media for business communication [11], customer communication [12] and managing business processes or the internal way of working [7], research on social media in organizations is still scarce. 


\subsection{Theoretical Background of BPM}

Various BPM attempts explain the lifecycle through which each business process evolves. Although BPM lifecycles differ in the naming and number of phases, they closely relate to the established Plan-Do-Check-Act cycle [13]. This means that each business process should first be identified and modeled or designed ("PLAN") before it can be deployed or executed ("DO"), monitored and analyzed ("CHECK") in order to be improved, optimized or innovated ("ACT"). Only few BPM lifecycles include a "MANAGEMENT" phase around the PDCA. BPM lifecycles are increasingly criticized for being technology-oriented and neglecting the organizational success factors [14, 15]. Consequently, a more holistic view on BPM, which takes into account the organizational culture and structure, is called "Business Process Orientation" (BPO) $[16,17]$. For instance, de Bruin and Rosemann [18] developed a maturity model including six capability areas (or critical success factors): (1) methods, (2) IT, (3) governance, (4) strategic alignment, (5) people, and (6) culture. Other holistic BPM scholars focused on one particular area, e.g. process-oriented values in the "culture" area [15]. A comprehensive overview of BPM capability areas is provided by Van Looy et al. [19], based on a theoretical validation in the literature and existing theories and an empirical validation based on $69 \mathrm{BPM} / \mathrm{BPO}$ maturity models. This BPM framework consists of six main capability areas with 17 sub capabilities: (A) process modeling, (B) process deployment, (C) process optimization, (D) process management, (E) a process-oriented culture, and $(\mathrm{F})$ a process-oriented structure. The first three areas relate to the PDCA cycle, while the fourth considers the managerial aspects per business process. The final two areas cover organizational success factors, and transform BPM to BPO.

The capability areas presented above should be adopted based on their contingency with an organization's business context to reach an optimal level [14, 20]. Other researchers cover dynamic capabilities to achieve process changes [21]. The latter coexist with the BPM capability areas, since process changes will be realized by changing one or more (operational) capability areas. Since our focus is rather on critical success factors for the state of BPM than on the change procedure itself, this study focuses on operational capability areas for BPM [19]. To our knowledge, no study digs deeper into the benefits of social media for BPM or an organization's process orientation with all its capability areas and subareas [24, 25].

\section{Methodology}

The systematic literature review (SLR) is "a form of secondary study that uses a welldefined methodology to identify, analyze and interpret all available evidence related to a specific research question in a way that is unbiased and (to a degree) repeatable" [22, p. 7]. It typically follows a protocol [23] (Table 1). 
Table 1. The Systematic Literature Review protocol for this study.

\begin{tabular}{|c|c|}
\hline Protocol elements & Translation to this study \\
\hline $\begin{array}{l}\text { Research objective and } \\
\text { questions }\end{array}$ & $\begin{array}{l}\text { What is the state of research on social media in the BPM discipline? } \\
\text { (To be answered by SLR-RQ1 and SLR-RQ2) }\end{array}$ \\
\hline Sources searched & $\begin{array}{l}\text { Emerald, IEEE Explore, Science Direct, Scopus, Web of Science, } \\
\text { AIS Electronic Library and ACM Digital Library }\end{array}$ \\
\hline Search terms & $\begin{array}{l}\text { Social media, business process*, (business) process management, } \\
\text { (management) information systems }\end{array}$ \\
\hline Search strategy & $\begin{array}{l}\text { Peer-reviewed journals and conference papers; theoretical and } \\
\text { empirical studies; no publication date limit, no sector limit, no topic } \\
\text { limit; search terms contained in articles' title, abstract and } \\
\text { keywords }\end{array}$ \\
\hline Inclusion criteria & Business processes or BPM and social media implementation \\
\hline Exclusion criteria & $\begin{array}{l}\text { (a) Articles using "process*" with a different meaning than BPM } \\
\text { (b) Articles without full access }\end{array}$ \\
\hline Quality criteria & $\begin{array}{l}\text { (a) Only peer-reviewed articles in the academic databases chosen } \\
\text { (b) Following a validated and comprehensive BPM framework } \\
\text { [19] }\end{array}$ \\
\hline
\end{tabular}

\subsection{SLR Research Questions}

We supplemented the social media classification by Kietzmann et al. [10] with a validated framework that expands business processes to BPM and BPO [19].

- SLR-RQ1: In which particular BPM matters (i.e. conventional areas and subareas of BPM) is social media use most frequently investigated?

- SLR-RQ2: Considering SLR-RQ1, what are the research avenues and business implications of social media use in BPM, as mentioned in the literature?

\subsection{SLR Search and Selection Procedure}

Figure 1 illustrates our procedure to select papers for our research objective.

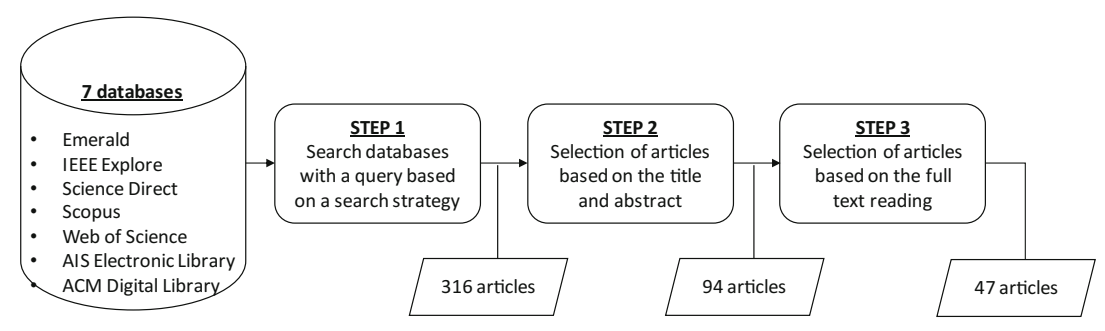

Fig. 1. The selection of articles considered for this research.

Step 1 was completed by browsing research papers in seven databases (Fig. 1). We considered all results until mid-2016, which is when the research started. To determine 
the link of the articles with our SLR-RQs, we decided to look for precise terms contained in the articles' title, abstract and keywords. Thus, our search was founded on combinations of: "social media" AND "business process*"; "social media" AND "business process management"; "social media" AND "process management"; "social media" AND "information systems"; and "social media" AND "management information systems". After Step 1, we came out with 316 papers.

In Step 2, we established inclusion and exclusion criteria that would help us determine which of the 316 articles were really relevant for our objective [22]. We decided to exclude all papers that used the terms "processing" or "to process" with a different meaning. For instance, based on their abstracts, we excluded every study that merely referred to a "diffusion process", "research process", "word processing", "sense making process" or "learning process". Every paper that simultaneously discussed business processes or BPM and social media would be included. Articles that we could not fully access were likewise discarded (i.e. six in total), resulting in 94 articles.

In Step 3, based on a full text reading, the previous criteria were applied to verify whether the articles were closely related to the social media implementation in BPM. A paper was only selected when it was related to at least one of the BPM categories that we considered with the applied framework [19]. Hence, we sampled 47 articles which can be found here: https://docs.google.com/document/d/1_N-8cyZSVLLrMUxSBujdd37y6d15BnZbX5wK07kB0Dw/edit?usp=sharing.

\subsection{SLR Classification}

We adopted a framework that offers a general overview of BPM, and which differentiates the narrow view on the traditional BPM lifecycle from the holistic view of business process orientation (BPO) by describing different layers of capability areas in clusters (i.e. BPM versus BPO), main areas and subareas [19]. Since the BPM framework and its capability areas were (theoretically and empirically) validated before, we classified the articles based on their understanding in previous research.

As part of the main capability area (A) "Process modeling”, we distinguished articles that explored: (1) "Business process design": papers specifying the relationship between events, activities and decisions in a value chain, as well as the actors involved and the related chains. (2) "Business process analysis": papers referring to the validation, simulation and verification of the designed business process models.

The main capability area (B) "Process deployment" included studies dedicated to: (3) "Business process implementation and enactment": papers including the operational models, implemented procedures and software systems. (4) "Business process measurement and control": papers referring to data collection and monitoring of running process instances for correcting deviations and providing status updates.

The main capability area (C) "Process optimization" embraced articles that examined: (5) "Business process evaluation": papers that intent to quantify the performance of finished process instances and the operational environment. (6) "Business process improvement": papers for making business processes conform to their process models and optimizing or innovating the models through redesign. 
The main capability area (D) "Process management" contained studies that focused on: (7) "Strategy and Key performance indicators": papers aligning business processes to strategic objectives and customer needs. (8) "External relationships and Service level agreements": papers actively involving external parties, like partnering with suppliers and customers. (9) "Roles and responsibilities": papers discussing the process manager and his/her team responsible for the performance and improvements of a specific business process. (10) "Skills and training": papers elaborating on the acquisition of skills for the actors involved. (11) "Daily management": papers dealing with specific management domains to be executed by the process manager.

The main capability area (E) "Process-oriented culture" was represented by articles that aimed at organizational characteristics, instead of a specific business process: (12) "Process-oriented values": papers presenting values which facilitate the realization of the previous capability areas (e.g. customer focus, empowerment, innovation, multidisciplinary collaboration and trust). (13) "Process-oriented attitudes and behaviors": papers discussing attitudes and behaviors that facilitate BPM across business processes and so concretize the defined values, such as BPM awareness, knowledge sharing and acceptance of change. (14) "Process-oriented appraisals and rewards": papers related to the HR implications (e.g. combining team incentives with individual benchmarks related to process performance). (15) "Top management commitment": papers in which top managers also support BPM and create a process-related C-level leadership role with responsibilities.

The main capability area (F) "Process-oriented structure" likewise comprised articles focusing on the organization characteristics, yet with more structural interests: (16)

"Process-oriented organization chart": papers determining changes in the organization structure to emphasize the cross-departmental business processes and the new roles. (17) "Process-oriented bodies": papers creating governance bodies across business processes, e.g. a BPM program management council and competence center.

\section{Results}

\subsection{Results for SLR-RQ1}

Figure 2 shows that most conventional BPM capability areas in the framework by Van Looy et al. [19] were covered by the sampled papers, except for the "Process-oriented structure" area. The attention of current studies was most frequently attained by the utility of social media in the "Process management" and "Process-oriented culture" areas of BPM (i.e. covered by respectively 37 and 30 sampled papers), followed by the areas related to the traditional BPM lifecycle (i.e. 28 papers for "Process optimization", 26 for "Process modeling" and 24 for "Process deployment").

Figure 3 refines Fig. 2 by illustrating differences among the BPM subareas. The "External relationships and Service Level Agreements" and the "Process-oriented attitudes and behaviors" were the most frequently captured practices in the reviewed literature (i.e. both with 17 papers). They are closely followed by the subareas of "Business process design" (i.e. 16 papers), "Business process implementation and enactment" (i.e. 


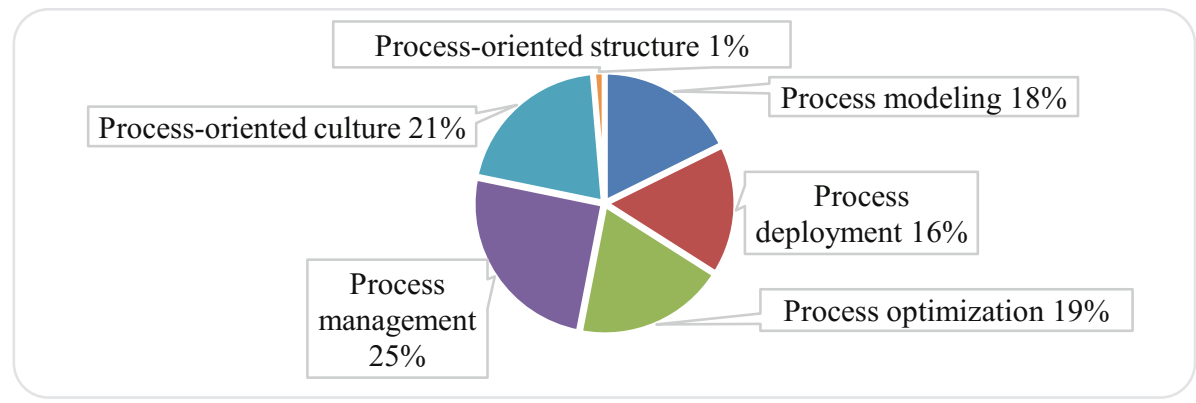

Fig. 2. The relative importance of the articles classified per BPM capability area ( $N=47)$.

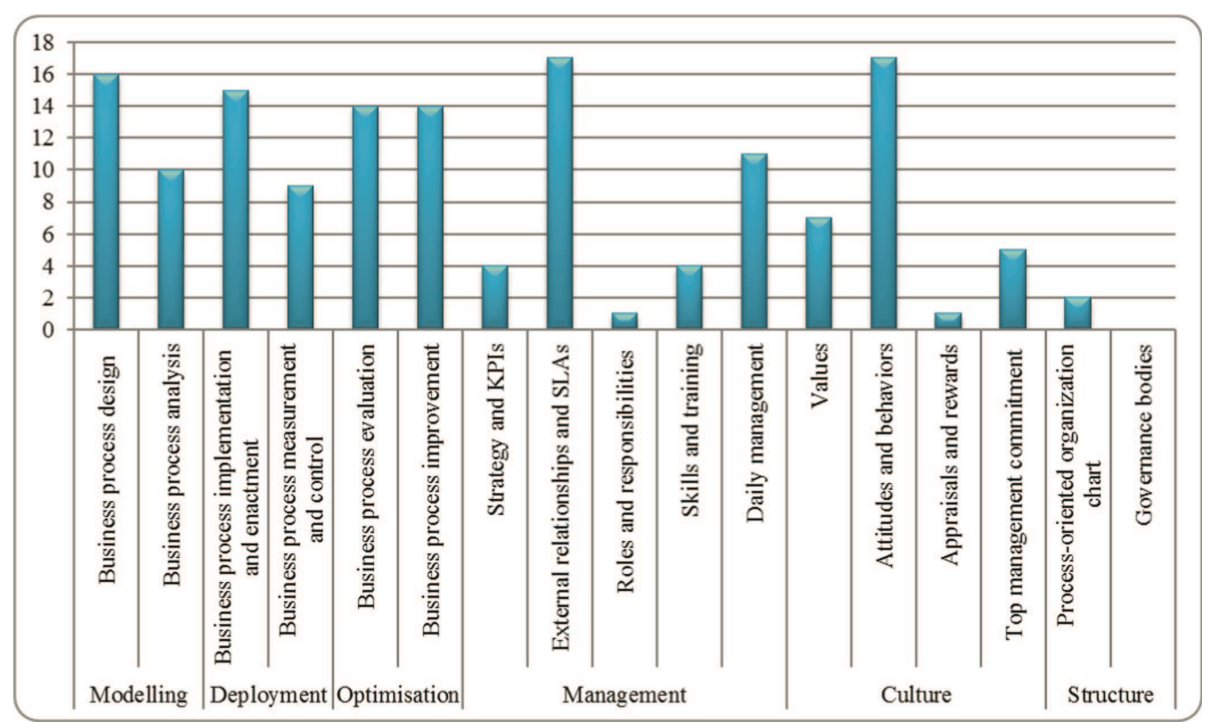

Fig. 3. The articles classified across BPM capability areas and subareas $(\mathrm{N}=47)$.

15 papers), "Business process evaluation" (i.e. 14 papers) and "Business process improvement" (i.e. 14 papers) in the traditional BPM lifecycle.

\subsection{Results for SLR-RQ2}

A qualitative analysis of the sampled papers in Nvivo allowed us to obtain an overview of the most frequently used terms related to the social media implementation in a business context (Fig. 4). The word clouds were obtained by browsing the frequency of keywords specifically describing the relationship between BPM and social media per BPM capability area. The full texts of the 47 papers, including references, were analyzed for this purpose. Given the fact that slight differences in wordings were observed for the areas related to the BPM lifecycle, we summarized them as PDCA. 


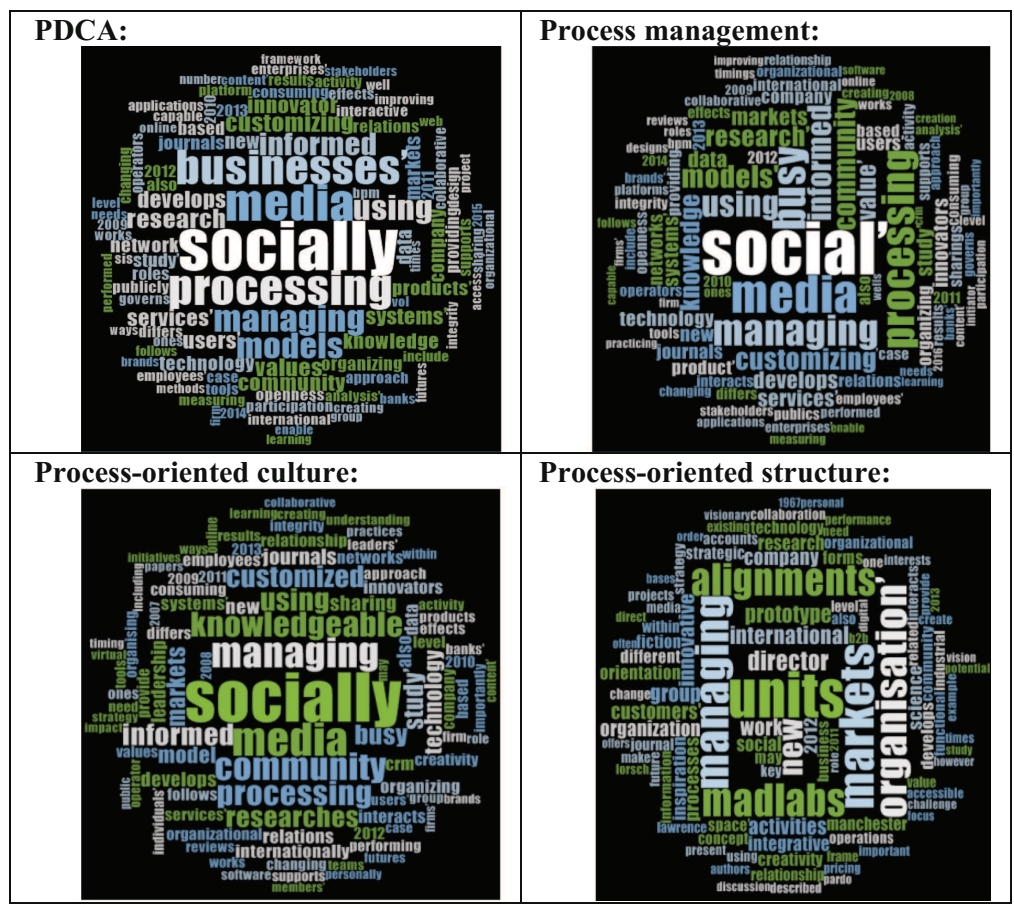

Fig. 4. The resulting word cloud per BPM capability area $(\mathrm{N}=47)$.

In addition to the word clouds, we had a closer look at the word frequency queries to distinguish typical terms and gain more insight per BPM capability area (Table 2). The most recurrent terms are shown in Table 2; a list that could be further extended taking into account all terms that have been used to describe the objective.

Table 2. The word frequency query results per BPM capability area $(\mathrm{N}=47)$.

\begin{tabular}{|c|c|}
\hline BPM capability area & Frequently used terms typifying each area (word count) \\
\hline PDCA & $\begin{array}{l}\text { "Processing" (1781); "Models" (1197); "Customizing" (785); } \\
\text { "Innovator" (668); "Network" (560); "Openness" (411); } \\
\text { "Supports" (399); "Consuming" (392); "Platform" (331) }\end{array}$ \\
\hline Process management & $\begin{array}{l}\text { "Managing" (1487); "Busy” (1366); “Customizing" (1177); } \\
\text { "Community" (1073); "Informing" (977) }\end{array}$ \\
\hline Process-oriented culture & $\begin{array}{l}\text { "Managing" (1422); "Community" (1271); "Knowledgeable" } \\
\text { (1119); "Study" (816); "Sharing" (588); "Leadership" (507); } \\
\text { "Values" (338); "Learning" (271) }\end{array}$ \\
\hline Process-oriented structure & $\begin{array}{l}\text { "Units" (238); "Markets" (187); "Managing" (180); } \\
\text { "Organization" (167); “Alignments" (159); "New" (120); } \\
\text { "Director" (85); "Integrative" (54) }\end{array}$ \\
\hline
\end{tabular}




\section{Discussion}

With respect to the conventional BPM areas, our study shows most recurrent attention for social media use in the "Process management" and "Process-oriented culture" areas. One explanation is that social media can improve communication, which is fundamental for the external relationships of an organization (e.g. involving contact with partners and customers) and for the process-oriented attitudes and behaviors (e.g. focusing on knowledge sharing and acceptance of change). Nonetheless, communication is likewise a necessity for process modeling, deployment and optimization. The latter BPM areas encompass activities and decisions regarding the procedures and systems implemented in a business process as well as the evaluation and optimization of model performance, requiring updated information and continuous communication.

Current academic research seems centered on the more general or managementrelated aspects (e.g. culture-related attitudes and behaviors, and the external relationships with customers, suppliers and other stakeholders), which are less specific to BPM. The more technical aspects of business processes in the BPM lifecycle (e.g. collaborative process modeling, execution and optimization with partners) are considered to a somewhat lesser extent, but also appear to be attractive for researchers so far. Thus, one of our main findings is the need to improve an organization's relationships with customers, suppliers, stakeholders and employees. This can more easily and effectively be done through social media by recognizing all implications presented in Kietzmann's honeycomb [10] (Sect. 2). Such implications could be used individually or combined, and their relevance is that social media can help organizations recognize and better understand their audience and engagement needs.

As a refinement to SLR-RQ1, SLR-RQ2 looked at any difference in focus when applying social media in a business process context. In the traditional process lifecycle, social media platforms and networks appear to be mainly advised to create openness for modeling, deploying and optimizing business processes. For instance, openness may refer to better serving the customer, and possibly includes the customization or tailoring of products and services. Furthermore, openness is also linked to opportunities for innovation. Sampled papers suggested that social media could be suitable to include customers in the planning process or for idea generation. Co-creation and crowdsourcing are likewise found to be useful strategies, counting on people's willingness to help for a project to be developed. Comments systems enable knowledge and information sharing by idea competition, innovation contests, ratings and reviews.

In the "Management" area, social media are ways to facilitate an informed community of process participants and to support customization. The sample alludes to the necessity of an interactive relationship with customers through social networks and social CRM. Forms to accomplish relationship management proficiency could be web characteristics: ease of networking, ease of participation, ease of collaboration.

The sampled articles dealing with a "Process-oriented culture" applied social media to better share knowledge or best practices across the organization. Studies suggested the use of communication and coordination tools, organization memory tools and project management tools, e.g. online fora, wikis and various intranet or communication software that enable multi-point conferencing, text chat, and file transfers. 
Finally, the few papers on a "Process-oriented structure" mentioned the use of social media to align business units and markets, and to facilitate the coordination of processrelated activities across all value chains throughout the organization by a central director. The idea is to create new units whose specialty would be social media management and the coordination of activities among internal processes and at different levels. As such, a better alignment between business units is expected.

With respect to the honeycomb [10], the functions and purposes within the capability areas of an individual business process (i.e. "PDCA" and "Process management" in Table 2) mainly relate to the blocks of customer "identity", "presence", "relationships" and "reputation". Such BPM areas include activities for creating, monitoring, controlling and managing the value chains and the related communication flows of especially external relationships. The more organizational areas of "Process-oriented culture" and "Process-oriented structure" are closely linked to the honeycomb blocks of (internal) "conversations", "sharing" and "groups". These BPM areas typically affect the entire organization by enabling internal communication and information sharing, and an enhanced contact between organizational groups and communities.

Thus, by comparing our results with the social media contributions proposed by the honeycomb [10], we anticipate how social media could be implemented in a certain BPM capability area to appreciate and enhance the social media functions (Table 3).

Table 3. Recommendations for using social media per BPM capability area.

\begin{tabular}{|c|c|}
\hline BPM capability area & Examples of social media opportunities \\
\hline 1/Process modeling & $\begin{array}{l}\text { - Collaborative process modeling with partners } \\
\text { - Online requirements gathering with customers and stakeholders } \\
\text { - Co-creation and crowdsourcing for Research \& Development }\end{array}$ \\
\hline 2/Process deployment & $\begin{array}{l}\text { - E-commerce by mobile or tablet applications } \\
\text { - Streamlining information flows among employees and partners } \\
\text { - Online monitoring of product/service feedback } \\
\text { - Online complaint handling }\end{array}$ \\
\hline 3/Process optimization & $\begin{array}{l}\text { - Creating value from online customer feedback and complaints } \\
\text { - Collecting (improvement or innovation) ideas based on } \\
\text { gamification techniques among employees (e.g. the number of } \\
\text { bottom-up ideas, likes, badges, progress bars) }\end{array}$ \\
\hline 4/Process management & $\begin{array}{l}\text { - Incorporating social media in business process strategies } \\
\text { - Internal trainings on how social media and BPM can be combined } \\
\text { - Collaborative process management and social media } \\
\text { management } \\
\text { - Social CRM and customer process management }\end{array}$ \\
\hline 5/Process-oriented culture & - Using wikis or the Intranet to share knowledge and best practices \\
\hline $\begin{array}{l}\text { 6/Process-oriented } \\
\text { structure }\end{array}$ & $\begin{array}{l}\text { - Creating a competence center (or Center of Excellence) with } \\
\text { expertise on synergies between BPM, social media and BI/big data } \\
\text { - Formal coordination among business processes (e.g. by wikis) }\end{array}$ \\
\hline
\end{tabular}

Finally, we give recommendations to attain the implications derived from our study. First, we revealed a lack of research regarding social media in the area of "Processoriented structure". This gap should empirically be investigated to verify whether 
managers do not recognize a necessity for social media in this area or whether another reason prevails. Moreover, managerial awareness about social media opportunities should be increased through informative workshops or seminars, since a number of executives do not truly understand their benefits and implications [10]. Furthermore, instead of focusing on one or a few BPM areas, the relationships inside and outside an organization can be improved by combining social media tools through different BPM areas. Such an omnichannel management strategy could provide seamless communication and control of business processes, anytime, from anyplace, with real-time visibility of systems, processes and people. More recommendations are given in Table 3.

\section{Conclusion}

This article focused on gaps in the literature and their practical implications, taking into account the limitations inherent to our SLR protocol. Although the current literature covers different sectors and topics to which BPM is related, many opportunities still seem to exist to further scrutinize the implications on BPM in more detail.

First, the literature on social media for BPM is increasing, but it is still relatively limited. Secondly, given the multidisciplinary approach that is inherent to our research subject, possible theories to underpin future BPM work might belong to disciplines such as communication science, psychology, management and business economics (e.g. marketing, innovation), and information systems. We particularly think of relationship management, innovation strategies, gamification theories, or social network techniques using big data. Thirdly, social media may provide more diverse opportunities (e.g. crowdsourcing and gamification) to organizations than the ones mostly covered in the investigated body of knowledge. Hence, future research could likewise consider social software as an alternative to the currently contemplated social media keyword. Next, we call for more research on omnichannel management strategies to let BPM evolve by social media in a more comprehensive manner, i.e. by examining how different business contexts profit from new technologies and digital innovation across the BPM capability areas to create synergies.

In the future, a more detailed analysis of the sampled papers could be enriching. An alternative starting point could be a smaller sample of articles from reputable journals and conferences only, based on more specific parameters to be reviewed. Another aim could be to look for BPM-related gaps in case studies to detect new ways for expanding BPM by social media implementation and, as such, improving an organization's business performance. Lastly, differentiating social media applications for BPM in large, medium-sized and smaller organizations could also be enriching.

\section{References}

1. Blanchard, O.: Social Media ROI. Pearson Education, Boston (2011)

2. Van Looy, A.: Social Media Management. Springer, Switzerland (2016)

3. Mangold, W.G., Faulds, D.J.: Social media: the new hybrid element of the promotion mix. Bus. Horiz. 52(4), 357-365 (2009) 
4. Kaplan, A.M., Haenlein, M.: Users of the world, unite! The challenges and opportunities of social media. Bus. Horiz. 53(1), 59-68 (2010)

5. Safko, L., Brake, D.K.: The Social Media Bible. John Wiley \& Sons Inc., Hoboken, New Jersey (2009)

6. Greenberg, P.: The impact of CRM 2.0 on customer insight. J. Bus. Ind. Mark. 25(6), 410419 (2010)

7. Choudhury, M.M., Harrigan, P.: CRM to social CRM. J. Strateg. Mark. 22(2), 149-176 (2014)

8. Cook, N.: Enterprise 2.0. Gower Publishing, Ltd., Aldershot (2008)

9. Helms, R.W., Booij, E., Spruit, M.R.: Reaching out: Involving users in innovation tasks through social media. Reach. Out 5, 15-2012 (2012)

10. Kietzmann, J.H., Hermkens, K., McCarthy, I.P., Silvestre, B.S.: Social media? Get serious! Bus. Horiz. 54(3), 241-251 (2011)

11. Smits, M., Mogos, S.: The impact of social media on business performance. In: ECIS, p. 125 (2013)

12. Fuduric, M., Mandelli, A.: Communicating social media policies. J. Commun. Manag. 18(2), 158-175 (2014)

13. Dumas, M., La Rosa, M., Mendling, J., Reijers, H.A.: Fundamentals of Business Process Management. Springer, Heidelberg (2013)

14. vom Brocke, J., Schmiedel, T., Recker, J., Trkman, P., Mertens, W., Viaene, S.: Ten principles of good business process management. Bus. Process Manag. J. 20(4), 530-548 (2014)

15. vom Brocke, J., Sinnl, T.: Culture in business process management: a literature review. Bus. Process Manag. J. 17(2), 357-377 (2011)

16. McCormack, K., Johnson, W.C.: Business Process Orientation. St. Lucie Press, Boca Raton, Florida (2001)

17. Van Looy, A., De Backer, M., Poels, G.: Defining business process maturity. Total Qual. Manag. Bus. Excell. 22(11), 1119-1137 (2011)

18. de Bruin, T., Rosemann, M.: Using the Delphi technique to identify BPM capability areas. In: ACIS Proceedings, vol. 42, pp. 642-653 (2007)

19. Van Looy, A., Backer, M.D., Poels, G.: A conceptual framework and classification of capability areas for business process maturity. Enterp. Inf. Syst. 8(2), 188-224 (2014)

20. vom Brocke, J., Zelt, S., Schmiedel, T.: On the role of context in business process management. Int. J. Inf. Manag. 36(3), 486-495 (2016)

21. Pöppelbuß, J., Plattfaut, R., Ortbach, K., Niehaves, B.: A dynamic capability-based framework for business process management: theorizing and empirical application. In: HICCS Proceedings, pp. 4287-4296 (2012)

22. Kitchenham, B.: Guidelines for performing systematic literature reviews in software engineering. Technical report, Ver. 2.3 EBSE (2007)

23. Boell, S.K., Cecez-Kecmanovic, D.: On being 'systematic' in literature reviews in IS. J. Inf. Technol. 30(2), 161-173 (2015)

24. Nascimento, A.M., da Silveira, D.S.: A systematic mapping study on using social media for business process improvement. Comput. Hum. Behav. 73, 670-675 (2017). https://doi.org/ 10.1016/j.chb.2016.10.016. (in press)

25. Erol, S., Granitzer, M., Happ, S., Jantunen, S., Jennings, B., Johannesson, P., Koschmider, A., Nurcan, S., Rossi, D., Schmidt, R.: Combining BPM and social software: contradiction or chance? J. Softw. Maint. Evol. Res. Pract. 22(6-7), 449-476 (2010) 